\title{
The importance of drug-induced sedation endoscopy (D.I.S.E.) techniques in surgical decision making: conventional versus target controlled infusion techniques - a prospective randomized controlled study and a retrospective surgical outcomes analysis
}

\author{
Andrea De Vito ${ }^{1}\left(D \cdot\right.$ Vanni Agnoletti $^{3} \cdot$ Gianluca Zani $^{4} \cdot$ Ruggero Massimo Corso $^{2}$ • \\ Giovanni D'Agostino $^{1} \cdot$ Elisabetta Firinu $^{1}$. Chiara Marchi $^{1} \cdot$ Ying-Shuo Hsu $^{5}$. \\ Stefano Maitan $^{2} \cdot$ Claudio Vicini $^{1}$
}

Received: 19 October 2016 / Accepted: 27 December 2016 / Published online: 17 February 2017

(C) Springer-Verlag Berlin Heidelberg 2017

\begin{abstract}
Drug-Induced Sedation Endoscopy (DISE) consists of the direct observation of the upper airways during sedative-induced sleep, allowing the identification of the sites of pharyngeal collapse, which is the main pathological event in Obstructive Sleep Apnea (OSA). The Authors have compared Target Controlled Infusion (TCI) sedation endoscopy (TCI-DISE) technique to conventional DISE (CDISE), performed by a manual bolus injection of sedative agent, to recreate accurately and safely snoring and apnea patterns comparable to natural sleep. The authors conducted a prospective, randomized, long-term study and a retrospective analysis of surgical outcomes. The apneaevent observation and its correlation with pharyngeal collapse patterns is the primary endpoint; secondary endpoints are defined as stability and safety of sedation plan of DISE-TCI technique. From January 2009 to January 2011, OSA patients were included in the study and randomly allocated into two groups: the bolus injection conventional DISE group and the TCI-DISE group. Third endpoint is to compare the surgical outcomes enrolling OSA patients
\end{abstract}

Andrea De Vito

dr.andrea.devito@gmail.com

1 Otorhinolaryngology and Oral Surgery Unit, Department of Head and Neck Surgery, Morgagni-Pierantoni Hospital, Forlì, Italy

2 Anaesthesia and Intensive Care Unit, Emergency Department, G.B. Morgagni-Pierantoni Hospital, Forlì, Italy

3 Anaesthesia and Intensive Care Unit, Emergency Department, M. Bufalini Hospital, Cesena, Italy

4 Anesthesia and Intensive Care Unit, Santa Maria delle Croci Hospital, Ravenna, Italy

5 Department of Otolaryngology, Shin Kong Wu Ho-Su Memorial Hospital, Taipei, Taiwan, Republic of China from January 2009 to June 2015. We recorded the complete apnea-event at oropharynx and hypopharynx levels in $15 / 50$ pts in conventional DISE group (30\%) and in 99/123 pts in TCI-DISE group $(81 \%)(p<0.0001)$. Four pts needed oxygen in conventional DISE group because a severe desaturation occurred during the first bolus of propofol $(1 \mathrm{mg} /$ $\mathrm{kg})(p=0.4872 \mathrm{~ns})$. We recorded instability of the sedation plan in 13 patients of conventional DISE group (65\%) and 1 patient of the TCI-DISE group (5\%) $(p=0.0001)$. In 37 TCI-DISE group surgical patients we reported a significant reduction of postoperative AHI (from $42.7 \pm 20.2$ to $11.4 \pm 10.3$ ) in comparison with postoperative $\mathrm{AHI}$ in 15 C-DISE group surgical patients (from $41.3 \pm 23.4$ to $20.4 \pm 15.5)(p=0.05)$. Our results suggest the DISE-TCI technique as first choice in performing sleep-endoscopy because of its increased accuracy, stability and safety. However, it is mandatory an accurate assessment of PSG/PM, which allows us to differentiate OSA patients in whom UA anatomical abnormalities are predominant in comparison with not-anatomical pathophysiologic factors, achieving good surgical patient's selection and outcomes as a consequence.

Keywords Sedation endoscopy - Obstructive sleep apnea $\cdot$ Target controlled infusion $\cdot$ Surgical outcomes

\section{Introduction}

The obstruction of upper airways due to the collapse of the pharyngeal walls (apnea-event) is the main pathological event observed in patients suffering from Obstructive Sleep Apnea (OSA), which represents one of the most common Sleep Breathing Disorders (SBD), affecting up to $9 \%$ of men and $4 \%$ of women worldwide [1]. Snoring is 
associated with apnea and usually arises as unique symptom before the onset of the OSA, but during peripheral apneic events, snoring usually precedes and follows the pharyngeal collapse. The most frequent sites of upper airways collapse are at pharyngeal level (soft palate, lateral pharyngeal walls, tonsils, base of the tongue), but increasing data reported in the literature underlie a significant role of larynx during apneic events, where primary (at epiglottic site in most of cases) or secondary (by means of base of the tongue hypertrophy) obstructions during apneic events can be observed [2].

Understanding the sites of upper airways collapse and its patterns is of paramount importance during diagnostic decision-making process, moreover, for referring the patient to the surgical treatment, and remain an open question in this field of research. Up to now fiber-optic nasopharyngeal endoscopy represents the main diagnostic tool performed in OSA patients, but it is usually carried out in awake patient, in a clinic setting, different from the spontaneous sleep situations in which apneas normally happen. During the awake fiber-optic nasopharyngeal endoscopy, the Muller maneuver (forced inspiratory suction with mouth and nose closed) has been usually performed. It allows an estimation of different patterns of pharyngeal collapse, but it presents several pitfalls: skill of the patient performing the maneuver and experienced skill of the operator analyzing the pharyngeal collapse patterns. Moreover, it is not possible observe clearly a primary laryngeal obstruction and only suspect a secondary laryngeal obstruction [3].

In 1991, Croft and Pringle [4, 5] first proposed a drug-induced sedation endoscopy (DISE), which allows the direct observation of the upper airways during sedative-induced sleep. Up to now, the main sedative agents employed during DISE are propofol and/or midazolam (used alone or in combination each other) and different doses have been considered and administered by means of bolus injection [6], conventional DISE technique (C-DISE), or by means of Target Controlled Infusion pump (TCI) (TCI-DISE) [7]. TCI system has been developed to provide improved convenience and control during intravenous anesthesia. The basic principle consists of setting and adjusting the target blood concentration-and depth of anesthesia-as required on clinical grounds. TCI technology uses different pharmacokinetic modeling to control the infusion rate of the pump, which allows a direct control of the sedative agent brain concentration rather than its blood concentration.

In 2010, we published our preliminary results of a prospective randomized controlled study, where conventional DISE technique in OSA patients has been compared with an effect-site (cerebral) TCI-DISE technique, to provide a more standardized and controlled state of sedation and recreate snoring and apnea patterns in the best possible way to simulate a spontaneous sleep situation [8]. In this paper, the Authors reported more consistent results of the prospective randomized controlled study and a retrospective analysis of surgical outcomes obtained, comparing the effectiveness, safety and stability of TCI-DISE technique with Conventional DISE in evaluation of OSA patients during diagnostic decision-making process and its impact in surgical outcomes.

\section{Materials and methods}

These were a prospective randomized controlled study and a retrospective analysis, performed in the Head and Neck Department, Ear Nose and Throat (ENT) Unit of Morgagni-Pierantoni Hospital, Forlì, Italy. The studies were approved by the local ethics committee and were designed and conducted in compliance with the principles of Good Clincal Practice regulations and the Helsinki declaration. Informed and written consent was obtained from patients before inclusion in these studies.

After the first prospective randomized controlled study, comparing the conventional bolus injection DISE technique with the DISE-TCI technique, published in 2010 [8], a more consistent patients' population has been enrolled and studied by means of the two techniques up to January 2011. We also selected candidates for referring to surgical treatment, allowing us to realize a retrospective analysis of surgical outcomes obtained in the patients' population who underwent surgical treatment from 2009 to 2015.

A computer-generated schedule created the random assignments for the C-DISE and TCI-DISE groups and the results were sealed in numbered envelopes.

The primary outcome is to investigate the effectiveness of the TCI-DISE technique in a more consistent patients' population, looking for a complete and stable sequence of snoring-obstructing hypo/apnea-oxygen desaturation-breathing (observation window), with good observation of levels and its repeatability during the same exam in the same patient, and correlate it with collapse patterns of upper airways. We considered a complete apnea/snoring cycle when snoring precedes and follows the apneic event [9]. The second outcome is to confirm the stability and safety of the TCI-DISE sedation plan, evaluating incremental modifications of BIS and variation of oxygen saturation $>3$ during the procedure. The third outcome is to analyze the surgical outcomes, comparing pre- and post-operative AHI scores as a result of the surgical procedures carried out on the surgical patients, selected by means of the two DISE techniques. The fourth outcome is to compare the differences of pharyngeal and laryngeal patterns observed during the awake endoscopic evaluation performing Mueller 
maneuver, with the pharyngeal and laryngeal patterns observed during the both DISE techniques applied. For this purpose, we analyzed the pre-operative awake NOHL patterns' score to pre-operative NOHL patterns' score observed during the conventional bolus injection DISE (C-DISE group) and TCI-DISE (TCI-DISE group). We excluded from the analysis the nose, because of the absence of significant modification of nasal cavities during sedation state.

\section{Inclusion/exclusion criteria}

According to the American Sleep Association (ASA) [10], we enrolled OSA patients, with a Apnea-Hypopnea Index (AHI) of 15-30, evaluated by unattended polygraphy (eight channels polygraphy: $\mathrm{SpO} 2$, HR, Flow cannula sensor, Actometer, Abdominal and Thoracic external effort-sensor, EOG; MAP Poly-MESAM, Medicair, Milano, Italy) [11]. Other inclusion criteria were: men and women, age 18-65 years, Body Mass Index (BMI) $<30$, awake oxygen saturation $>95 \%$ and able to read and sign the consent form.

Exclusion criteria were: Chronic Obstructive Pulmonary Disease (COPD), liver disease (Child Pugh 1-3), history of chronic use of sedatives, narcotics, alcohol or illicit drugs, history of 1st and 2nd degree heart block (not paced), left ventricular Ejection Fraction (EF) $<50$, allergy to propofol or to any medications, OSHAS surgical failure patients and pregnant women.

\section{Study design}

For the prospective randomized controlled study all sleep endoscopy was carried out in the operating theatre setting, with patients in their ward bed in a supine position and with a comfortable ambient temperature. Before DISE, we performed an awake fiber-optic nasopharyngeal endoscopy with the Muller maneuver.

\section{N.O.H.L. classification}

Grade and patterns of upper airways collapse were evaluated at the nasal cavities (Nose), retro-palatal space (Oropharynx) and base of the tongue space (Hypopharynx). We defined a total collapse of pharyngeal walls during Muller maneuver as grade 4 , a collapse $<75 \%$ as grade 3, a collapse $<50 \%$ as grade 2 , a collapse $<25 \%$ as grade 1 , no collapse during Muller maneuver as grade 0 . We used the same classification for the nasal cavities from 4 to 0 , if we found the presence of obstructive nasal septum deviation, inferior turbinate hypertrophy or other nasal obstructive pathology. We also defined the different patterns of pharyngeal collapse as: (1) transversal (t), if we observed a lateral pharyngeal collapse; (2) anterior-posterior (ap), if we observed a collapse due to anterior pharyngeal collapse and (3) concentric (c), if we observed a lateral plus anterior-posterior pharyngeal walls collapse pattern. We eventually recorded the presence of different laryngeal anatomical obstruction, especially at epiglottic level, defined as positive or negative collapse.

In this way it was possible to define the several sites and patterns of nasal obstruction and pharyngeal collapse in a simple manner, N.0-4, O.0-4c,t,a-p, H.0-4c,t,a-p, (see Table 1) [12].

In addition to a starting upper airways evaluation, the clinical assessment of the patients included: heart rate, noninvasive blood pressure (NIBP), oxygen saturation ( $\mathrm{SpO} 2$ ). To analyze the deep of sedation we applied the bispectral index (BIS) monitoring. Bispectral index (BIS) is a scale derived from cerebral electrical activity to measure the effect of specific anesthetic drugs on the brain. The recommended range in anesthesia guided by BIS is 40-60 [13].

\section{Conventional bolus injection DISE group (C-DISE group)}

A sleep endoscopy was manually performed using $1 \mathrm{mg} \mathrm{kg}{ }^{-1}$ of propofol as induction bolus and $20 \mathrm{mg}$ of boluses was repeated every $2 \mathrm{~min}$, with a $20-\mathrm{ml}$ syringe.

Table 1 N.O.H.L. classification

\begin{tabular}{lllll}
\hline Site & Nose & Oropharynx & Hypopharynx & Larynx \\
\hline Obstruction \% grade (value) & $0-25 \%: 1$ & & \\
& $25-50 \%: 2$ & & \\
& $50-75 \%: 3$ & & \\
& $75-100 \%: 4$ & & & \\
Collapse \% grade (value) & & $0-25 \%: 1$ & $0-25 \%: 1$ & Positive \\
& & $25-50 \%: 2$ & $25-50 \%: 2$ & Negative \\
& & $50-75 \%: 3$ & $50-75 \%: 3$ & \\
\hline
\end{tabular}


We performed the C-DISE technique from January 2009 to January 2011.

\section{Target controlled infusion system DISE group (TCI-DISE group)}

Sleep endoscopy was performed by a Target Controlled Infusion system (Alaris ${ }^{\circledR}$ PK Syringe Pump) using Schnider model in effect-site (cerebral) targeted infusion 50-ml prefilled syringe of $1 \%$ propofol. Schnider system is a complex pharmacokinetic/pharmacodynamic (PK/PD) model that allows to obtain different rates of drug from the values of age, height, weight and lean body mass of the patient [14]. The starting dose of propofol was $1.5 \mathrm{mcg} \mathrm{ml}^{-1}$ and increments of $0.2 \mathrm{mcg} \mathrm{ml}^{-1}$ took place when the new cerebral concentration of propofol was reached. In this way, we realized a slow technique of TCI propofol infusion. We performed the TCI-DISE technique from January 2009 up to January 2015.

During both procedures, we recorded vital parameters and modifications of N.O.H.L. classification when the new cerebral concentration of propofol was reached and before the next injection of propofol. We performed a complete upper airways evaluation, including nasal cavities during first awake examination, whereas we assessed the modification at Oropharyngeal, Hypopharyngeal and Laryngeal sites during DISE, because of the absence of significant modification of nasal cavities during sedation state.

All the sleep endoscopies were performed by the same group of anesthesiologists (AV, GZ, MC) and the same otorhinolaryngologist (ADV). In each group we recorded the apnea-event and its correlation with the mean value of BIS and other vital parameters (SpO2, NIBP, HR) every 2 min.

An evaluation of postoperative AHI has been performed by means of unattended polygraphy at minimum of 6 months after surgical treatment.

\section{Statistical analysis}

The statistical analysis was performed by SPSS software version 18 (IBM Corp. Somers, NY, USA). All enrolled patients were included in analysis. The Fisher's Exact Test was used to compare snoring-obstructing hypo/apnea-oxygen desaturation-breathing (observation window), the stability of the sedation plan and the safety of the procedures. Mann-Whitney test was used to estimate the difference in propofol consumption between two groups; Dunn's Multiple Comparison test to match BIS and saturation values in C-DISE and TCI-DISE groups. Paired $t$ test was used to compare pre-operative AHI to post-operative AHI obtained after surgical treatment in each DIS group.

\section{Results}

\section{Prospective randomized controlled study}

A total of 205 consecutive OSA patients scheduled for the DISE participated in the prospective controlled randomized study. 11/205 patients refused to participate, 21/205 did not meet the inclusion criteria (14/205 for BMI $>30$ and 7/205 for awake $\mathrm{SpO} 2<95 \%$ ). 173 eligible patients were enrolled in the study, with a mean AHI of $22 \pm 7$ and a mean value of $\mathrm{SpO} 2$ during polygraphy of $92 \% \pm 8.50 .50$ (43 male) patients were allocated in C-DISE group whereas 123 (112 male) in TCI-DISE group. Mean patients age in C-DISE group was $48 \pm 12$ years with range from 36 to 60 . Mean patients age in TCI-DISE group was $51 \pm 11$ years with range from 40 to 62 .

We recorded a $30 \%$ of complete and stable sequence of snoring-obstructing hypo/apnoea-oxygen desaturationbreathing with good observation of levels at oropharynx and hypopharynx levels in C-DISE group and 80\% in TCI DISE-group $(15 / 50$ versus $99 / 123-P<0.001)$. These results were confirmed by the blinded revision conducted by the head of Department in $100 \%$ of patients. We reported an instability of the sedation plan, represented by incremental modifications of BIS and variation of oxygen saturation $>3$, occurring in the same period of time, in $65 \% \mathrm{C}$-DISE group and in 5\% TCI-DISE group (32 versus $6-P=0.0001)$. No patient required oxygen administration before sedation or in TCI-DISE group during the procedure, in comparison with $8 / 50$ pts (16\%) in C-DISE group, who needed oxygen because of a severe desaturation as a consequence of the first bolus $1 \mathrm{mg} \mathrm{kg}^{-1}$ of propofol $(P<0.05)$; no patient had complications as nausea, vomiting, moderate-severe hypotension or ECG modifications. The mean propofol consumption was $132 \pm 32 \mathrm{mg}$ in C-DISE group corresponding to $148 \mathrm{mg} \pm 37$ in TCI-DISE group $(P<0.05)$ (see Table 2$)$.

The mean value of BIS in C-DISE group during the total performance-time of sleep endoscopy was $73 \pm 18$ and $78 \pm 13$ in TCI-DISE group $(P=0.001)$. TCI-DISE group

Table 2 DISE intraopertive summary. C-group versus T-group

\begin{tabular}{llll}
\hline Events & C-group & T-group & $P$ value \\
\hline Apneic events & $13 / 50(30 \%)$ & $99 / 123(80 \%)$ & $P<0.001$ \\
Instability Plan sedation & $32 / 50(65 \%)$ & $6 / 123(5 \%)$ & $P=0.0001$ \\
Safety & $48 / 50$ & $123 / 123$ & $P<0.05$ \\
Propofol consumption & $132 \pm 32 \mathrm{mg}$ & $148 \mathrm{mg} \pm 37$ & $P<0.05$ \\
mean value of BIS & $73 \pm 18$ & $78 \pm 13$ & $P=0.001$ \\
Mean value of SpO2 & $91.47 \pm 5$ & $89.89 \pm 6$ & $P=0.001$ \\
Mean value of duration & $6.4 \pm 3.2 \mathrm{~min}$ & $14.9 \pm 4.7 \mathrm{~min}$ & $P<0.001$ \\
$\quad$ & & & \\
\hline
\end{tabular}


and C-DISE group mean values $\mathrm{SpO} 2$ is, respectively, $91.47( \pm 5)$ and $89.89( \pm 6)(P=0.001)$. The mean value of duration time for sedation endoscopic procedures, intended as pts in operating theatre and ready to perform the exam, was $6.4 \pm 3.2 \mathrm{~min}$. for C-DISE group and $14.9 \pm 4.7 \mathrm{~min}$. for TCI-DISE group $(P<0,001)$ (see Table 2$)$.

\section{Retrospective surgical study}

We selected a total number of 53 patients for surgical treatment: 15 male patients from C-DISE group and 37 from TCI-DISE group (female: 2) (see Tables 3, 4) from 2009 to 2015. No statistical differences have been reported between the mean preoperative AHI of C-DISE group $(41.3 \pm 23.4)$ and the mean preoperative AHI of TCI-DISE group $(42.7 \pm 20.2)$. Tables 3 and 4 show a detailed schedule of all surgical procedures carried out in both group of patients.
We reported a significant reduction of postoperative AHI (from $42.7 \pm 20.2$ to $11.4 \pm 10.3$ ) in TCI-DISE group, in comparison with postoperative AHI in 15 C-DISE group surgical patients (from $41.3 \pm 23.4$ to $20.4 \pm 15.5$ ) $(P=0.05)$. (see Tables 3, 4).

\section{Discussion}

Several DISE techniques are reported in the literature, with an increasing modification about how to perform the sedation, the main indications for DISE, and how to report DISE findings. Moreover, various issues regarding DISE validity and reliability are reported in the literature, especially if the sleep architecture during DISE is reproducible and comparable to that in natural sleep [15-23].

Table 3 C-DISE

\begin{tabular}{|c|c|c|c|c|c|c|c|}
\hline PT & Sex & BMI & N.O.H.L awake & NOHL DISE & $\begin{array}{l}\text { AHI pre- } \\
\text { operative }\end{array}$ & Surgical procedures & $\begin{array}{l}\text { AHI } \\
\text { post- } \\
\text { operative }\end{array}$ \\
\hline 1 & M & 27.78 & $\mathrm{~N} 2 \mathrm{O} 4 \mathrm{cH} 1 \mathrm{tL} 0$ & O4cH3apL1 Trap door epiglottidis & 26 & $\begin{array}{l}\text { TORS-BOT + EPIGLOTTOPLASTY + TRA- } \\
\text { CHEO + UP3 + NASAL SURGERY }\end{array}$ & 13 \\
\hline 2 & $\mathrm{~F}$ & 22.66 & N3O3cH2apL0 & O4cH4apL1 Trap door epiglottidis & 26.7 & $\begin{array}{l}\text { TORS-BOT + EPIGLOTTOPLASTY + TRA- } \\
\text { CHEO + LATERAL PHARYNGO- } \\
\text { PLASTY + NASAL SURGERY }\end{array}$ & 16.9 \\
\hline 3 & M & 20.98 & $\mathrm{~N} 3 \mathrm{O} 3 \mathrm{cH} 3 \mathrm{tLO}$ & $\mathrm{O} 4 \mathrm{cH} 4 \mathrm{tLO}$ & 30 & MAXILLOMANDIBULAR ADVANCEMENT & 7 \\
\hline 4 & M & 26.12 & N1O3tH2apL0 & $\mathrm{O} 3 \mathrm{tH} 3 \mathrm{tLO}$ & 16 & $\begin{array}{l}\text { LATERAL PHARYNGOPLASTY + TONSIL- } \\
\text { LECTOMY + NASAL SURGERY + HYOID } \\
\text { SUSPENSION }\end{array}$ & 1.5 \\
\hline 5 & M & 31.79 & $\mathrm{~N} 1 \mathrm{O} 3 \mathrm{tH} 2 \mathrm{tL0}$ & $\mathrm{O} 4 \mathrm{tH} 3 \mathrm{tLO}$ & 30 & $\begin{array}{l}\text { UP3 + TONSILLECTOMY + NASAL SUR- } \\
\text { GERY + HYOID SUSPENSION }\end{array}$ & 4 \\
\hline 6 & M & 24.34 & $\mathrm{~N} 3 \mathrm{O} 3 \mathrm{cH} 2 \mathrm{tLO}$ & $\mathrm{O} 4 \mathrm{cH} 3 \mathrm{tLO}$ & 23 & $\begin{array}{l}\text { LATERAL PHARYNGOPLASTY + NASAL } \\
\text { SURGERY + HYOID SUSPENSION }\end{array}$ & 5 \\
\hline 7 & M & 32.79 & $\mathrm{~N} 2 \mathrm{O} 4 \mathrm{cH} 2 \mathrm{tLO}$ & O4cH3apL0 & 27.3 & $\begin{array}{l}\text { TORS-BOT + EPIGLOTTOPLASTY + TRA- } \\
\text { CHEO + UP3 + NASAL SURGERY }\end{array}$ & 8.2 \\
\hline 8 & M & 29.32 & $\mathrm{~N} 2 \mathrm{O} 3 \mathrm{cH} 4 \mathrm{apL} 0$ & O3cH4apL1 Trap door epiglottidis & 19 & $\begin{array}{l}\text { LATERAL PHARYNGOPLASTY + TONSIL- } \\
\text { LECTOMY + NASAL SURGERY + HYOID } \\
\text { SUSPENSION }\end{array}$ & 5 \\
\hline 9 & M & 22.72 & $\mathrm{~N} 3 \mathrm{O} 2 \mathrm{cH} 2 \mathrm{tLO}$ & O3cH4apL0 & 30 & $\begin{array}{l}\text { TORS-BOT + EPIGLOTTOPLASTY + TRA- } \\
\text { CHEO + LATERAL PHARYNGO- } \\
\text { PLASTY + TONSILLECTOMY + NASAL } \\
\text { SURGERY }\end{array}$ & 7.9 \\
\hline 10 & M & 31.73 & $\mathrm{~N} 2 \mathrm{O} 4 \mathrm{cH} 3 \mathrm{tLO}$ & O4cH3apL0 & 30 & MAXILLOMANDIBULAR ADVANCEMENT & 7 \\
\hline 11 & M & 26.88 & $\mathrm{~N} 2 \mathrm{O} 3 \mathrm{cH} 2 \mathrm{cLO}$ & O4cH3apL0 & 30 & $\begin{array}{c}\text { TORS-BOT + EPIGLOTTOPLASTY + TRACHE- } \\
\text { OPHARYNGOPLASTY + NASAL SURGERY }\end{array}$ & 10 \\
\hline 12 & M & 26.12 & N3O3cH3apL0 & O4cH3apL0 & 30 & PHARYNGOPLASTY + NASAL SURGERY & 20 \\
\hline 13 & M & 30.12 & $\mathrm{~N} 1 \mathrm{O} 4 \mathrm{cH} 4 \mathrm{cLO}$ & O4cH4cL1 Trap door epiglottidis & 30 & $\begin{array}{l}\text { TORS-BOT + EPIGLOTTOPLASTY + TRA- } \\
\text { CHEO + UP3 + TONSILLECTOMY }\end{array}$ & 8 \\
\hline 14 & M & 25.70 & N3O3cH2apL0 & O4cH2apL1 Trap door epiglottidis & 30 & $\begin{array}{l}\text { TORS-BOT + EPIGLOTTOPLASTY + TRA- } \\
\text { CHEO + UP3 + TONSILLECTOMY + TURBI- } \\
\text { NOPLASTY }\end{array}$ & 4.5 \\
\hline 15 & M & 31.80 & $\mathrm{~N} 2 \mathrm{O} 3 \mathrm{cH} 2 \mathrm{apL} 0$ & O4cH3apL1 Trap door epiglottidis & 29 & $\begin{array}{l}\text { TORS-BOT + EPIGLOTTOPLASTY + TRA- } \\
\text { CHEO + UP3 + TONSILLECTOMY }\end{array}$ & 10 \\
\hline
\end{tabular}


Table 4 TCI-DISE group

\begin{tabular}{|c|c|c|c|c|c|c|c|}
\hline PT & Sex & BMI & NOHL awake & NOHL DISE & $\begin{array}{l}\text { AHI pre- } \\
\text { operative }\end{array}$ & Surgical procedure & $\begin{array}{l}\text { AHI } \\
\text { post- } \\
\text { operative }\end{array}$ \\
\hline 1 & M & 25.2 & $\mathrm{~N} 2 \mathrm{O} 2 \mathrm{cH} 2 \mathrm{tLO}$ & O4cH3apL1 Trap door epiglottidis & 30 & $\begin{array}{l}\text { TORS-BOT + EPIGLOTTO- } \\
\text { PLASTY + TRACHEO + NASAL } \\
\text { SURGERY + LATERAL PHARYNGO- } \\
\text { PLASTY + TONSILLECTOMY }\end{array}$ & 17 \\
\hline 2 & M & 17.5 & N2O1H2apL0 & O4cH3apL0 & 29.8 & $\begin{array}{l}\text { TORS-BOT + TRACHEOLATERAL } \\
\text { PHARYNGOPLASTY + TONSILLEC- } \\
\text { TOMY + NASAL SURGERY }\end{array}$ & 10 \\
\hline 3 & $\mathrm{~F}$ & 30 & $\mathrm{~N} 3 \mathrm{O} 3 \mathrm{cH} 3 \mathrm{tLO}$ & $\mathrm{O} 4 \mathrm{cH} 3 \mathrm{tLO}$ & 30 & $\begin{array}{l}\text { LATERAL PHARYNGO- } \\
\text { PLASTY + TONSILLEC- } \\
\text { TOMY + NASAL SURGERY + HYOID } \\
\text { SUSPENSION }\end{array}$ & 9 \\
\hline 4 & M & 27.1 & $\mathrm{~N} 3 \mathrm{O} 3 \mathrm{cH} 2 \mathrm{tLO}$ & $\mathrm{O} 4 \mathrm{cH} 4 \mathrm{tLO}$ & 29 & $\begin{array}{l}\text { LATERAL PHARYNGO- } \\
\text { PLASTY + TONSILLEC- } \\
\text { TOMY + NASAL SURGERY + HYOID } \\
\text { SUSPENSION }\end{array}$ & 12 \\
\hline 5 & M & 26.1 & $\mathrm{~N} 3 \mathrm{O} 2 \mathrm{cH} 2 \mathrm{tLO}$ & $\mathrm{O} 3 \mathrm{cH} 3 \mathrm{tLO}$ & 30 & $\begin{array}{l}\text { LATERAL PHARYNGO- } \\
\text { PLASTY + TONSILLEC- } \\
\text { TOMY + NASAL SURGERY + HYOID } \\
\text { SUSPENSION }\end{array}$ & 13.5 \\
\hline 6 & M & 25.9 & $\mathrm{~N} 3 \mathrm{O} 1 \mathrm{H} 4 \mathrm{cLO}$ & $\mathrm{O} 3 \mathrm{cH} 4 \mathrm{tLO}$ & 25 & $\begin{array}{l}\text { LATERAL PHARYNGO- } \\
\text { PLASTY + TONSILLEC- } \\
\text { TOMY + NASAL SURGERY + HYOID } \\
\text { SUSPENSION }\end{array}$ & 17.1 \\
\hline 7 & M & 23.7 & $\mathrm{~N} 2 \mathrm{O} 4 \mathrm{cH} 2 \mathrm{tLO}$ & $\mathrm{O} 4 \mathrm{cH} 3 \mathrm{apL} 1$ omega fashion epiglottidis & 30 & $\begin{array}{l}\text { TORS-BOT + EPIGLOTTO- } \\
\text { PLASTY + TRACHEO + NASAL } \\
\text { SURGERY + LATERAL PHARYNGO- } \\
\text { PLASTY + TONSILLECTOMY }\end{array}$ & 8.1 \\
\hline 8 & M & 21.5 & $\mathrm{~N} 3 \mathrm{O} 3 \mathrm{cH} 3 \mathrm{apL0}$ & $\mathrm{O} 4 \mathrm{cH} 4 \mathrm{apL0}$ & 29 & $\begin{array}{l}\text { TORS-BOT + TRACHEO + NASAL } \\
\text { SURGERY + LATERAL PHARYNGO- } \\
\text { PLASTY + TONSILLECTOMY }\end{array}$ & 15.2 \\
\hline 9 & M & 30 & $\mathrm{~N} 0 \mathrm{O} 2 \mathrm{cH} 2 \mathrm{tLO}$ & $\mathrm{O} 3 \mathrm{cH} 4 \mathrm{tLO}$ & 28 & $\begin{array}{l}\text { LATERAL PHARYNGO- } \\
\text { PLASTY + TONSILLEC- } \\
\text { TOMY + NASAL SURGERY + HYOID } \\
\text { SUSPENSION }\end{array}$ & 11 \\
\hline 10 & M & 26.87 & $\mathrm{~N} 3 \mathrm{O} 3 \mathrm{cH} 3 \mathrm{apL0}$ & O4cH3apL1 Trap door epiglottidis & 30 & $\begin{array}{l}\text { TORS-BOT + EPIGLOTTO- } \\
\text { PLASTY + TRACHEO + NASAL } \\
\text { SURGERY + LATERAL PHARYNGO- } \\
\text { PLASTY + TONSILLECTOMY }\end{array}$ & 11 \\
\hline 11 & M & 29.76 & $\mathrm{~N} 2 \mathrm{O} 2 \mathrm{cH} 2 \mathrm{tLO}$ & $\mathrm{O} 3 \mathrm{cH} 3 \mathrm{tLO}$ & 18 & $\begin{array}{l}\text { LATERAL PHARYNGO- } \\
\text { PLASTY + NASAL SUR- } \\
\text { GERY + HYOID SUSPENSION }\end{array}$ & 4 \\
\hline 12 & M & 29.87 & $\mathrm{~N} 1 \mathrm{O} 2 \mathrm{cH} 2 \mathrm{apL0}$ & O3cH3tL1 secondary epiglottidis collapse & 30 & $\begin{array}{l}\text { TORS-BOT + EPIGLOTTO- } \\
\text { PLASTY + TRACHEO + NASAL } \\
\text { SURGERY + LATERAL PHARYNGO- } \\
\text { PLASTY + TONSILLECTOMY }\end{array}$ & 15 \\
\hline 13 & M & 23.57 & $\mathrm{~N} 3 \mathrm{O} 1 \mathrm{cH} 2 \mathrm{apL0}$ & O3cH3apL1 Trap door epiglottidis & 30 & $\begin{array}{l}\text { TORS-BOT + EPIGLOTTO- } \\
\text { PLASTY + TRACHEO + NASAL } \\
\text { SURGERY + LATERAL PHARYNGO- } \\
\text { PLASTY + TONSILLECTOMY }\end{array}$ & 10 \\
\hline 14 & M & 24.5 & $\mathrm{~N} 3 \mathrm{O} 3 \mathrm{cH} 2 \mathrm{apL0}$ & O4cH3apL1 Trap door epiglottidis & 26 & $\begin{array}{l}\text { TORS-BOT + EPIGLOTTO- } \\
\text { PLASTY + TRACHEO + NASAL } \\
\text { SURGERY + LATERAL PHARYNGO- } \\
\text { PLASTY + TONSILLECTOMY }\end{array}$ & 6 \\
\hline
\end{tabular}


Table 4 (continued)

\begin{tabular}{|c|c|c|c|c|c|c|c|}
\hline $\mathrm{PT}$ & Sex & BMI & NOHL awake & NOHL DISE & $\begin{array}{l}\text { AHI pre- } \\
\text { operative }\end{array}$ & Surgical procedure & $\begin{array}{l}\text { AHI } \\
\text { post- } \\
\text { operative }\end{array}$ \\
\hline 15 & M & 26.7 & $\mathrm{~N} 2 \mathrm{O} 2 \mathrm{cH} 2 \mathrm{tLO}$ & O3cH4apL1 Trap door epiglottidis & 29.8 & $\begin{array}{l}\text { TORS-BOT + EPIGLOTTO- } \\
\text { PLASTY + TRACHEO + NASAL } \\
\text { SURGERY + LATERAL PHARYNGO- } \\
\text { PLASTY + TONSILLECTOMY }\end{array}$ & 9 \\
\hline 16 & M & 23.77 & $\mathrm{~N} 3 \mathrm{O} 2 \mathrm{cH} 1 \mathrm{apL} 0$ & $\mathrm{O} 3 \mathrm{cH} 3 \mathrm{tL} 1$ secondary epiglottidis collapse & 30 & $\begin{array}{l}\text { TORS-BOT + EPIGLOTTO- } \\
\text { PLASTY + TRACHEO + NASAL } \\
\text { SURGERY + LATERAL PHARYNGO- } \\
\text { PLASTY + TONSILLECTOMY }\end{array}$ & 4 \\
\hline 17 & M & 26.5 & N3O3tH1tL0 & O4tH1tL0 & 26 & $\begin{array}{l}\text { LATERAL PHARYNGO- } \\
\text { PLASTY + TONSILLEC- } \\
\text { TOMY + NASAL SURGERY }\end{array}$ & 4 \\
\hline 18 & M & 26.4 & $\mathrm{~N} 3 \mathrm{O} 3 \mathrm{cH} 2 \mathrm{tLO}$ & O4cH3apL1 Trap door epiglottidis & 29 & $\begin{array}{l}\text { TORS-BOT + EPIGLOTTO- } \\
\text { PLASTY + TRACHEO + NASAL } \\
\text { SURGERY + LATERAL PHARYNGO- } \\
\text { PLASTY + TONSILLECTOMY }\end{array}$ & 10 \\
\hline 19 & M & 26.85 & $\mathrm{~N} 2 \mathrm{O} 3 \mathrm{cH} 2 \mathrm{apL0}$ & O4cH3apL1 Trap door epiglottidis & 29 & $\begin{array}{l}\text { TORS-BOT + EPIGLOTTO- } \\
\text { PLASTY + TRACHEO + NASAL } \\
\text { SURGERY + LATERAL PHARYNGO- } \\
\text { PLASTY + TONSILLECTOMY }\end{array}$ & 10 \\
\hline 20 & M & 23.67 & $\mathrm{~N} 3 \mathrm{O} 3 \mathrm{cH} 1 \mathrm{tL0}$ & $\mathrm{O} 4 \mathrm{cH} 2 \mathrm{tLO}$ & 27 & $\begin{array}{l}\text { LATERAL PHARYNGO- } \\
\text { PLASTY + TONSILLEC- } \\
\text { TOMY + NASAL SURGERY }\end{array}$ & 0 \\
\hline 21 & M & 30 & $\mathrm{~N} 3 \mathrm{O} 3 \mathrm{cH} 3 \mathrm{apL} 0$ & O4cH3apL1 Trap door epiglottidis & 19.8 & $\begin{array}{l}\text { TORS-BOT + EPIGLOTTO- } \\
\text { PLASTY + TRACHEO + NASAL } \\
\text { SURGERY + LATERAL PHARYNGO- } \\
\text { PLASTY + TONSILLECTOMY }\end{array}$ & 3.2 \\
\hline 22 & M & 29 & $\mathrm{~N} 3 \mathrm{O} 2 \mathrm{cH} 2 \mathrm{tLO}$ & O4cH3apL1 Trap door epiglottidis & 30 & $\begin{array}{l}\text { TORS-BOT + EPIGLOTTO- } \\
\text { PLASTY + TRACHEO + NASAL } \\
\text { SURGERY + LATERAL PHARYNGO- } \\
\text { PLASTY + TONSILLECTOMY }\end{array}$ & 10.08 \\
\hline 23 & M & 26.54 & $\mathrm{~N} 2 \mathrm{O} 2 \mathrm{cH} 1 \mathrm{tLO}$ & $\mathrm{O} 4 \mathrm{cH} 2 \mathrm{tL} 0$ & 24 & $\begin{array}{l}\text { LATERAL PHARYNGO- } \\
\text { PLASTY + TONSILLEC- } \\
\text { TOMY + NASAL SURGERY + HYOID } \\
\text { SUSPENSION }\end{array}$ & 4 \\
\hline 24 & M & 28.34 & $\begin{array}{l}\mathrm{N} 3 \mathrm{O} 2 \mathrm{cH} 2 \mathrm{tL} 1 \\
\text { Arytenoid } \\
\text { Edema }\end{array}$ & $\mathrm{O} 4 \mathrm{cH} 2 \mathrm{tL} 1$ arytenoid oedema & 26.6 & $\begin{array}{l}\text { LATERAL PHARYNGO- } \\
\text { PLASTY + TONSILLEC- } \\
\text { TOMY + NASAL SURGERY + CO2la- } \\
\text { ser arytenoidotomy }\end{array}$ & 6 \\
\hline 25 & M & 23.8 & $\mathrm{~N} 3 \mathrm{O} 2 \mathrm{cH} 1 \mathrm{tLO}$ & $\mathrm{O} 4 \mathrm{cH} 3 \mathrm{tLO}$ & 24 & $\begin{array}{l}\text { LATERAL PHARYNGO- } \\
\text { PLASTY + TONSILLEC- } \\
\text { TOMY + NASAL SURGERY + HYOID } \\
\text { SUSPENSION }\end{array}$ & 4 \\
\hline 26 & M & 26 & $\mathrm{~N} 2 \mathrm{O} 2 \mathrm{cH} 2 \mathrm{tLO}$ & O3cH3apL1 Trap door epiglottidis & 29 & $\begin{array}{l}\text { TORS-BOT + EPIGLOTTO- } \\
\text { PLASTY + TRACHEO + NASAL } \\
\text { SURGERY + LATERAL PHARYNGO- } \\
\text { PLASTY + TONSILLECTOMY }\end{array}$ & 10 \\
\hline 27 & M & 27 & $\mathrm{~N} 2 \mathrm{O} 3 \mathrm{cH} 2 \mathrm{tLO}$ & $\mathrm{O} 3 \mathrm{cH} 3 \mathrm{tLO}$ & 30 & $\begin{array}{l}\text { LATERAL PHARYNGO- } \\
\text { PLASTY + TONSILLEC- } \\
\text { TOMY + NASAL SURGERY + HYOID } \\
\text { SUSPENSION }\end{array}$ & 5 \\
\hline 28 & M & 28 & $\mathrm{~N} 2 \mathrm{O} 2 \mathrm{cH} 2 \mathrm{tLO}$ & $\mathrm{O} 3 \mathrm{cH} 3 \mathrm{tLO}$ & 24 & $\begin{array}{l}\text { LATERAL PHARYNGO- } \\
\text { PLASTY + TONSILLECTOMY + SEP- } \\
\text { TOTURBINOPLASTY + HYOID } \\
\text { SUSPENSION }\end{array}$ & 9 \\
\hline 29 & M & 30 & $\mathrm{~N} 3 \mathrm{O} 3 \mathrm{cH} 3 \mathrm{apL} 0$ & O4cH4apL0 & 30 & $\begin{array}{l}\text { MAXILLOMANDIBULAR ADVANCE- } \\
\text { MENT }\end{array}$ & 4 \\
\hline
\end{tabular}


Table 4 (continued)

\begin{tabular}{|c|c|c|c|c|c|c|c|}
\hline PT & Sex & BMI & NOHL awake & NOHL DISE & $\begin{array}{l}\text { AHI pre- } \\
\text { operative }\end{array}$ & Surgical procedure & $\begin{array}{l}\text { AHI } \\
\text { post- } \\
\text { operative }\end{array}$ \\
\hline 30 & M & 21.7 & $\mathrm{~N} 3 \mathrm{O} 2 \mathrm{cH} 1 \mathrm{apL0}$ & O4cH3apL1 Trap door epiglottidis & 30 & $\begin{array}{l}\text { TORS-BOT + EPIGLOTTO- } \\
\text { PLASTY + TRACHEO + NASAL } \\
\text { SURGERY + LATERAL PHARYNGO- } \\
\text { PLASTY + TONSILLECTOMY }\end{array}$ & 15 \\
\hline 31 & M & 27.1 & $\mathrm{~N} 3 \mathrm{O} 3 \mathrm{cH} 3 \mathrm{tLO}$ & $\mathrm{O} 4 \mathrm{cH} 3 \mathrm{tLO}$ & 25 & $\begin{array}{l}\text { LATERAL PHARYNGO- } \\
\text { PLASTY + TONSILLEC- } \\
\text { TOMY + NASAL SURGERY + HYOID } \\
\text { SUSPENSION }\end{array}$ & 5 \\
\hline 32 & M & 29 & $\mathrm{~N} 1 \mathrm{O} 2 \mathrm{cH} 1 \mathrm{tL0}$ & O4cH3apL1 Trap door epiglottidis & 30 & $\begin{array}{l}\text { TORS-BOT + EPIGLOTTO- } \\
\text { PLASTY + TRACHEO + NASAL } \\
\text { SURGERY + LATERAL PHARYNGO- } \\
\text { PLASTY + TONSILLECTOMY }\end{array}$ & 15 \\
\hline 33 & M & 24 & $\mathrm{~N} 2 \mathrm{O} 3 \mathrm{cH} 2 \mathrm{tLO}$ & O4cH3apL1 Trap door epiglottidis & 30 & $\begin{array}{l}\text { TORS-BOT + EPIGLOTTO- } \\
\text { PLASTY + TRACHEO + NASAL } \\
\text { SURGERY + LATERAL PHARYNGO- } \\
\text { PLASTY + TONSILLECTOMY }\end{array}$ & 1.1 \\
\hline 34 & $\mathrm{~F}$ & 25.5 & $\mathrm{~N} 3 \mathrm{O} 2 \mathrm{cH} 2 \mathrm{apL} 0$ & O3cH3apL1 Trap door epiglottidis & 30 & $\begin{array}{l}\text { TORS-BOT + EPIGLOTTO- } \\
\text { PLASTY + TRACHEO + NASAL } \\
\text { SURGERY + LATERAL PHARYNGO- } \\
\text { PLASTY + TONSILLECTOMY }\end{array}$ & 8.5 \\
\hline 35 & M & 25 & $\mathrm{~N} 3 \mathrm{O} 1 \mathrm{cH} 1 \mathrm{tL} 0$ & $\mathrm{O} 3 \mathrm{cH} 3 \mathrm{tLO}$ & 28 & $\begin{array}{l}\text { LATERAL PHARYNGO- } \\
\text { PLASTY + TONSILLEC- } \\
\text { TOMY + NASAL SURGERY + HYOID } \\
\text { SUSPENSION }\end{array}$ & 8 \\
\hline 36 & M & 23 & $\mathrm{~N} 2 \mathrm{O} 3 \mathrm{cH} 3 \mathrm{tLO}$ & $\mathrm{O} 4 \mathrm{cH} 3 \mathrm{tLO}$ & 18 & $\begin{array}{l}\text { LATERAL PHARYNGO- } \\
\text { PLASTY + TONSILLEC- } \\
\text { TOMY + NASAL SURGERY }\end{array}$ & 4 \\
\hline 37 & M & 24 & $\mathrm{~N} 2 \mathrm{O} 3 \mathrm{cH} 1 \mathrm{tL0}$ & $\mathrm{O} 4 \mathrm{cH} 2 \mathrm{tLO}$ & 22 & $\begin{array}{l}\text { LATERAL PHARYNGO- } \\
\text { PLASTY + TONSILLEC- } \\
\text { TOMY + NASAL SURGERY }\end{array}$ & 4 \\
\hline
\end{tabular}

(1) nasal surgery: including septoplasty with or without inferior turbinoplasty and/or splitting concha bullosa and/or FESS (2) TORS: Trans Oral Robotic Surgery (3) BOT: partial base of the tongue glossectomy

One of the main findings of our study is that DISE carried out by means of Target Controlled Infusion pump and a slow perfusion of propofol, allows us to reach a paraphysiological pattern of sleep and apneic events, associated with snoring. In our series of 123 patients we recorded a complete and stable sequence of snoringobstructing hypo/apnea-oxygen desaturation-breathing with good observation of levels (observation window) in $81 \%$ of patients, comparing with $30 \%$ of patients who had undergone conventional bolus injection DISE technique. The starting dose of propofol of $1.5 \mathrm{mcg} \mathrm{ml}^{-1}$ and the increasing dose of $0.2 \mathrm{mcg} \mathrm{ml}^{-1}$ carried out when the new cerebral concentration of propofol was obtained, allows us to reach a grade of sedation slowly, and this could be important in carrying out a paraphysiological pattern of sleep and a stable pattern of light-sedation. The TCI technique allows us to limit the rapid onset of propofol action and to limit the depth of sedation, observed in our study by BIS monitoring. We observed a mean BIS value of $78 \pm 13$ during the examination but recording a complete cycle of observation window.

Our findings obtained in C-DISE group show us that if DISE is carried out by means of conventional bolus injection technique a complete and stable sequence of snoringobstructing hypo/apnea-oxygen desaturation-breathing with good observation of levels (observation window) is reached in only $15 / 50$ patients $(30 \%)$ with mean BIS value of $73 \pm 18$. One possible explanation could be that in conventional DISE technique the fast sleep induction, suppressing the subjective response to sedative agent, determines in most of patients an excessive muscle relaxation and sudden pharyngeal collapse as a consequence, realizing false-positive patterns of upper airways collapse with loss of an effective observation window. Moreover, we recorded a rapid fall of BIS to deep values as 40 in most of patients, particularly at the beginning of the procedure, event that invalidated DISE examination. 
As general rule our data confirm the deeper BIS value is during DISE, the lesser the probability to obtain an effective cycle of observation window. Therefore, we strong suggest to apply always a BIS monitoring during DISE, maintaining its range between 75 and 65 during the procedure, obtaining a light sedation level status, consisting in loss of consciousness, defined as loss of response to verbal stimulation at a normal volume, comparable to a modified Ramsay sedation score of 5 [24].

TCI infusion maintains blood concentration at a set level and, therefore, the effective brain concentration, allowing the operator to modulate the depth of sedation at a right level to realize the dynamic modification of pharyngeal walls occurring during snoring and apnea events. TCI technique allows us to limit the rapid onset of propofol action and to limit the depth of sedation, observed in our study by BIS monitoring.

As general rule, we suggest to not consider any UA modification different from a complete and stable sequence of snoring-obstructing hypo/apnea-oxygen desaturationbreathing [25-27] and to consider more sleep studies and more respiratory tests in any patient, in whom it is not possible to visualize a good observation window during the light sedation stage, with the aim to identify the presence of a REM-related OSA, no-OSA SBD or overlap syndromes $[28,29]$.

The analysis of the secondary endpoints leads us to consider the TCI-DISE group more stable, because of the increased accuracy of the TCI system in maintaining the brain concentration of propofol at a steady-state. Manually infusion may cause an inadequate and unsettled brain concentration of the sedative agent.

More importantly in C-DISE group, eight patients had a severe desaturation during the first manual bolus injection ( $\mathrm{SpO} 261-65 \%)$ that represents a life-threatening situation and this could be avoided using the TCI technique (see Table 2).

These findings confirm our preliminary results [8] and allow us to state the role of TCI pump as essential technical equipment required in performing DISE.

A remarkable finding that we have confirmed in this study was the observation of apnea-events at multilevel sites in the upper airways in each group. No patient showed a single site of obstruction, even when the larynx contributed to the obstruction.

The main limitation of DISE is concerning the use of sedative agents, which does not allow to simulate the REM sleep stage, not allowing to analyze REM-related OSA as a consequence [29]. Other DISE limitations consist of the sleep-disturbing effect of the endoscope and the time wasted assessing the study. Two other relative limitations of the TCI technique are the necessity of the TCI pump and BIS in the operating theatre, two expensive but largely applied medical tools. Another limitation of the TCI system is the use of Schnider infusion protocol that has less accuracy for severe grades of BMI. Finally, it is important to take into account the longer time it takes to perform the DISE-TCI (15.2 $\pm 6 \mathrm{~min})$ which impacts the scheduling of the operating theatre time table.

Concerning retrospective surgical outcomes analysis literature data reported discordant surgical outcomes in patients selected by means of DISE. Koutsourelakis et al. assessed surgical outcomes in 41 OSA patients who underwent conventional bolus injection DISE, highlighting the importance of DISE in predicting the patients population with high probability to be responder or no-responder to surgical therapy, reporting a higher occurrence of complete or partial antero-posterior collapse at velum and of partial antero-posterior collapse at tongue base and epiglottis in responders compared to no-responders [30]. Vanderveken et al. underlined the predictive value of DISE performed by means of combined sedative agents (midazolam with a bolus injection of $1.5 \mathrm{mg}$ and/or with propofol using a TCI pump) in selecting surgical patient candidates for Implanted Upper Airway Stimulation devices. The Authors reported better outcomes with Upper Airways Stimulation in patients $(n=16)$ without palatal complete concentric collapse in comparison with patients with palatal anterior-posterior pattern of collapse [31]. Aktas et al. investigated the role of DISE (conventional bolus injection of propofol) in selecting patients for surgical treatment, reporting a significantly high operation success rate in the group of patients (14 cases) with obstruction of the upper airway according to DISE $(P<0.05)$, whereas a significantly low operation success rate in the group of patients ( 6 cases) with obstruction of the lower airways according to DISE $(P<0.01)$ [32].

Otherwise Blumen et al. in a retrospective study on surgical outcomes including 24 OSA patients, reported an overall surgical success rate in $58.3 \%$ of patients, but highlighting that, in 8 patients in the success group, DISE showed an obstruction site at hypopharyngeal level not treated by surgery. The authors stated that DISE could overestimate the sites of pharyngeal collapse in comparison with a more reliable awake clinical ENT examination in seated position [33].

The analysis of our surgical outcomes confirms a more effectiveness of TCI-DISE in evaluation of sites and pattern of pharyngeal wall, in comparison with C-DISE. In 37 TCI-DISE group surgical patients we reported a significant reduction of postoperative AHI (from $42.7 \pm 20.2$ to $11.4 \pm 10.3$ ) in comparison with postoperative AHI in $15 \mathrm{C}$-DISE group surgical patients (from $41.3 \pm 23.4$ to $20.4 \pm 15.5)(P=0.05)$. Moreover, our analysis in the surgical group of 53 patients showed a significant difference between awake NOHL patterns' 
score and DISE NOHL patterns' score $(P=0.05)$, particularly at hypopharyngeal and laryngeal site, allowing us to obtain significantly better surgical results (see Tables 3, 4).

In a recent systematic review on awake ENT examination versus DISE examination of OSAS patients, Certal et al confirm the direct impact of DISE on surgical decision making in OSA patients, in comparison with ENT awake examination, but up to now there no available published studies with evidence on the association between DISE impact and surgical outcomes [34].

Our results confirm the effectiveness of DISE in surgical decision making process and surgical outcomes. Even though we concur with review's Author conclusion, we strongly emphasize that if the majority of literature data were homogeneous in terms of minimum patients population required, DISE standardized technique, preoperative and postoperative AHI calculation, a more multicentric statistically consistent data of the impact of DISE in surgical outcomes could be easily achieved. Furthermore avoiding DISE bolus injection technique, deep BIS index, waiting for a reliable observation window could ensure us to have obtained a real paraphysiologic sleep condition and not a general anesthesia induction muscular relaxation. Certainly restricting our evaluation of OSA patients only to the awake conventional ENT examination with or without Muller's maneuver should be abandoned, for it demonstrated ineffectiveness as reported in the literature data [34]. In 2014, a first European Position Consensus Meeting on DISE reported a general consensus on indications required preliminary examinations, where to perform DISE, technical equipment required, staffing, local anesthesia, nasal decongestion, other medications, patient positioning, basics and special diagnostic maneuvers, drugs and observation windows and could represent an effort in achieving an effective standardization in DISE technique, with significant more appropriate patients' selection for surgical treatment [9].

The main limitation of DISE consists in its unlikelihood to clearly differentiate OSA patients in whom notanatomical pathophysiologic factors (U.A. muscular dysfunction, sleep-wake status and ventilator control instability and arousal threshold) are predominant to UA anatomical abnormalities [35] or differentiate OSA patients with associated respiratory diseases (Obese Hypoventilation Syndrome, Chronic Obstructive Pulmonary Disease, chest diseases, muscular neuropathy, ect.). We strongly recommend obtaining a clear Polysomnografic analysis, before performing DISE, which helps to avoid misunderstanding about DISE evaluation with unsuitable DISE conclusion and ineffective surgical treatments as a consequence.

\section{Conclusion}

In OSA patients, the identification of stable sequence of snoring-obstructing hypo/apnea-oxygen desaturationbreathing with good observation of levels (observation window) is of paramount importance for diagnostic accuracy and could significantly help the ENT surgeon in the choice of the most appropriate surgical technique to perform. Even in experienced hands, conventional DISE can potentially be ineffective and dangerous. Our results suggest that the DISE-TCI technique should be the first choice in performing sleep-endoscopy because of its increased accuracy, stability and safety, even though a clear polysomnografic analysis is imperative to allow us to differentiate OSA patients in whom UA anatomical abnormalities are predominant in comparison with not-anatomical pathophysiologic factors.

\section{Compliance with ethical standards}

Conflict of interest None.

\section{References}

1. Young T, Peppard PE, Gottlieb DJ (2002) Epidemiology of obstructive sleep apnea: a population health perspective. Am J Respir Crit Care Med 165:1217-1239

2. Viana Ada C (2015) Jr1, Thuler LC2, Araújo-Melo MH3. Druginduced sleep endoscopy in the identification of obstruction sites in patients with obstructive sleep apnea: a systematic review. Braz J Otorhinolaryngol 81(4): 439-446

3. Stuck BA, Maurer JT (2008 Dec) Airway evaluation in obstructive sleep apnea. Sleep Med Rev 12(6):411-436 (Epub 2007 Nov 28)

4. Croft CB, Pringle M (1991) Sleep nasendoscopy: a technique of assessment in snoring and obstructive sleep apnoea. Clin Otolaryngol Allied Sci 16(5):504-509

5. Pringle MB, Croft CB (1991) A comparison of sleep nasendoscopy and the Muller manoeuvre. Clin Otolaryngol Allied Sci 16:559-562

6. Kezirian EJ (2006) Drug-induced sleep endoscopy. Oper Tech Otolaryngol 17:230-232

7. Roblin G, Williams AR, Whittet H (2001) Target-controlled infusion in sleep endoscopy. Laryngoscope 111: 175-176

8. De Vito A, Agnoletti V, Berrettini S (2010) Drug-induced sleep endoscopy: conventional versus target controlled infusion techiques-a randomized controlled study. Eur Arch Otorhinolaryngol 268:457-462 [Epub published online(2011)]

9. De Vito A, Carrasco M, Agnoletti V et al (2014) European position paper on drug-induced sedation endoscopy (DISE). Sleep Breath 18(3):453-465. doi:10.1007/s11325-014-0989-6 (Epub 2014 May 26)

10. Ward Flemons W, Buysse D, Redline S et al (1999) Sleep-related breathing disordered in adults: recommendations for syndrome definition and measurement techniques in clinical research. The report of an American Academy of Sleep medicine Task Force. Sleep 22:667-689

11. Collop NA, Anderson WM, Boehlecke B et al (2007) Clinical guidelines for the use of unattended portable monitors in the 
diagnosis of obstructive sleep apnea in adult patients. Portable Monitoring Task Force of the American Academy of Sleep Medicine. J Clin Sleep Med 3:737-747

12. Vicini C, De Vito A, Benazzo M et al (2012) The nose oropharynx hypopharynx and larynx (NOHL) classification: a new system of diagnostic standardized examination for OSAHS patients. Eur Arch Otorhinolaryngol 269(4):1297-1300. doi:10.1007/ s00405-012-1965-z (Epub 2012 Feb 19)

13. Punjasawadwong $\mathrm{Y}$, Boonjeungmonkol N, Phongchiewboon A (2007) Bispectral index for improving anaesthetic delivery and postoperative recovery. Cochrane Database Syst Rev 17(4):CD003843

14. Absalom A, Struys MMRF ( 2007) An overview of TCI \& TIVA. 2nd edn. Academia Press, Belgium

15. Borowiecki B, Pollak CP, Weitzman ED et al (1978) Fibrooptic study of pharyngeal airway during sleep in patients with hypersomnia obstructive sleep-apnea syndrome. Laryngoscope 88:1310-1313

16. Sadaoka T, Kakitsuba N, Fujiwara Y et al (1996) The value of sleep nasendoscopy in the evaluation of patients with suspected sleep-related breathing disorders. Clin Otolaryngol Allied Sci 21:485-489

17. Berry S, Roblin G, Williams A et al (2005) Validity of sleep nasendoscopy in the investigation of sleep related breathing disorders. Laryngoscope 115:538-540

18. Hewitt RJ, Dasgupta A, Singh A et al (2009) Is sleep nasendoscopy a valuable adjunct to clinical examination in the evaluation of upper airway obstruction? Eur Arch Otorhinolaryngol 266:691-697

19. Rojewski TE, Schuller DE, Clark RW et al (1984) Videoendoscopic determination of the mechanism of obstruction in obstructive sleep apnea. Otolaryngol Head Neck Surg 92:127-131

20. Woodson BT, Wooten MR (1994) Comparison of upper-airway evaluations during wakefulness and sleep. Laryngoscope 104:821-828

21. Kotecha BT, Hannan SA, Khalil HM et al (2007) Sleep nasendoscopy: a 10-year retrospective audit study. Eur Arch Otorhinolaryngol 264:1361-1367

22. Quinn SJ, Daly N, Ellis PD (1995) Observation of the mechanism of snoring using sleep nasendoscopy. Clin Otolaryngol Allied Sci 20:360-364

23. Rodriguez-Bruno K, Goldberg AN, McCulloch CE et al (2009) Test-retest reliability of drug-induced sleep endoscopy. Otolaryngol Head Neck Surg 140:646-651
24. Ramsay MA, Savege TM, Simpson BR et al (1974) Controlled sedation with alphax-alone-alphadolone. Br Med J 2:656-659

25. Yu-Lun L, Yung-Lun N, Tsai-Yu W, Ting-Yu L et al (2015) Bispectral index in evaluating effects od sedation depth on druginduced sleep endoscopy. JCSM 11(9):1011-1020

26. Coppens M, Van Limmen JG, Schnider T et al (2010) Study of the time course of the clinical effect of propofol compared with the time course of the predicted effect-site concentration: performance of three pharmacokinetic-dynamic models. Br J Anaesth $104: 452-458$

27. Babar-Craig H, Rajani NK, Bailey P, Kotecha BT (2012) Validation of sleep nasendoscopy for assessment of snoring with bispectral index monitoring. Eur Arch Otorhinolaryngol 269:1277-1279

28. Subramani Y, Singh M, Wong J et al (2016) Understanding phenotypes of obstructive sleep apnea: applications in anesthesia, surgery and perioperative medicine. Anesth Analg 124(1):179-191

29. Beneto A, Soler-Algarra S, Salavert V (2016) 'REM-related OSA': a forgotten diagnostic? Possible path to under-diagnosing sleep apnea. Rev Neurol 63(11):481-487

30. Koutsourelakis I, Safiruddin F, Ravesloot M et al (2012) Surgery for obstructive sleep apnea: sleep endoscopy determinants of outcome. Laryngoscope 122(11):2587-2591

31. Vanderveken O, Maurer JT, Hohenhorst W et al (2013) Evaluation of drug-induced sleep endoscopy as a patient selection tool for implanted upper airway stimulation for obstructive sleep apnea. J Clin Sleep Med 9(5):433-438

32. Aktas O, Erdur O, Cirik AA, et al (2014) The role of druginduced sleep endoscopy in surgical planning for obstructive sleep apnea syndrome. Eur Arch Otorhinolaryngol doi:10.1007/ s00405-014-3162-8 (EpuB June)

33. Blumen MB, Latournerie V, Bequignon E et al (2015) Are the obstruction sites visualized on drug-induced sleep endoscopy reliable? Sleep Breath 19(3):1021-1026

34. Certal VF, Pratas R, Guimarães L et al (2016) Awake examination versus DISE for surgical decision making in patients with OSA: a systematic review. Laryngoscope 126(3):768-774

35. Eckert DJ, White DP, Jordan AS et al (2013) Defining phenotypic causes of obstructive sleep apnea. AM J Respir Crit Care Med 188(8):996-1004 